Supporting Information for

\title{
Parsimonious Potential Energy Surface Expansions Using Dictionary Learning with Multipass Greedy Selection
}

\author{
Daniel R. Moberg, Ahren W. Jasper,* and Michael J. Davis* \\ E-mail: ajasper@anl.gov (A.W.J.), davis@tcg.anl.gov (M.J.D.)
}

\section{Details of the multipass greedy method}

This section consists of four parts. The first part derives the leave-one-out crossvalidation (loocv) error, which is used in the paper. The second part describes the detailed schedules used for the multipass greedy algorithm of all cases presented in the paper and in the Supporting Information. The third part addresses the nature of the subsets used, presenting calculations which map out subsets that lie near one of the subsets generated with the multipass greedy algorithm. We end the Supporting Information with a note about a method for finding subsets not discussed in the main text.

The paper uses the loocv error as a predictive or out-of-sample error. The calculation of the loocv error starts with Eq. (3) in the text, which is repeated here and is used to calculate the expansion coefficients:

$$
c=\left(\mathbf{X}^{\mathrm{T}} \mathbf{X}\right)^{-1} \mathbf{X}^{\mathrm{T}} \mathbf{y}, \mathbf{y}=\mathbf{W V}, \mathbf{X}=\mathbf{W B}
$$

where the elements of $\mathbf{B}, B_{i j}$, describe the $j^{\text {th }}$ basis function evaluated at the $i^{\text {th }}$ point of the vector of potential values, $\mathbf{V}$. The matrix $\mathbf{W}$ is the diagonal weight matrix from Eq. (2) in the paper.

The weighted values of the potential energy, the y's, are related to the fitted weighted values of the potential energy by:

$$
\hat{\mathbf{y}}=\mathbf{H y}, \mathbf{H}=\mathbf{X}\left(\mathbf{X}^{\mathrm{T}} \mathbf{X}\right)^{-1} \mathbf{X}^{\mathrm{T}}
$$


The matrix $\mathbf{H}$ is commonly known as the hat matrix..$^{1-3}$ The Hat matrix can be used to define this out-of-sample relationship:2,3

$$
\mathrm{y}_{\mathrm{i}}-\widehat{\mathrm{y}}_{\mathrm{i}}^{(-\mathrm{i})}=\frac{\mathrm{y}_{\mathrm{i}}-\widehat{\mathrm{y}_{1}}}{1-\mathrm{h}_{\mathrm{ii}}}
$$

The $\mathrm{h}_{\mathrm{ii}}$ 's are the diagonal elements of the Hat matrix. The $\hat{y}_{i}^{(-i)}$ are the predicted values of the y's when the fitting is done with all the other points but that one. This leads to calculation of an out-of-sample error, commonly called the leave-one-out cross-validation error ("loocv" error): 3

$$
\begin{aligned}
& \mathrm{E}_{\text {loocv }}^{2}=\sum_{\mathrm{i}=1}^{\mathrm{M}}\left(\frac{\mathrm{y}_{\mathrm{i}}-\widehat{\mathrm{y}_{\mathrm{i}}}}{1-\mathrm{h}_{\mathrm{ii}}}\right)^{2} \\
& \mathrm{E}_{\text {loocv }}=\sqrt{\frac{\mathrm{E}_{\text {loocv }}^{2}}{\mathrm{M}}}
\end{aligned}
$$

The inset plot of Fig. 3b shows the ratio of this error to the rms error, which is defined in Eq. (2) in the paper. The rms error is a fitting error and the loocv error is a prediction error.

All multipass calculations in the paper were initiated from a subset of size one. This initial basis function was the one that gave the minimum rms error for the potential energy surface that was being fit. All multipass calculations in the paper used 100 passes in the forward direction and 100 passes in the backward direction $\left(n_{\mathrm{p}}=100\right)$. It was found from experience that the best way to find low error subsets using the multipass algorithm was to use several different path lengths, $n_{\mathrm{s}}$. Each set of multipass calculations has approximately $n_{\mathrm{p}}$ times the cost of a simple forward greedy calculation with a path length of $n_{\mathrm{s}}$. In addition, we ran many versions of the multipass calculations as noted in Table S1, adding to the overall cost. Computational costs were not found to be prohibitive, and so no effort was made to optimize the choices of $n_{\mathrm{p}}$ and $n_{\mathrm{s}}$. To err on the side of caution we ran many sets of choices as summarized in Tables S1 and S2. For example, 1-150 indicates 150 different passes of 100 were run with path lengths, $n_{\mathrm{s}}$, starting at 1 and ending with 150. As the basis sets got larger, we increased the maximum value of $n_{\mathrm{s}}$. 
For those values above 150 we incremented $n_{\mathrm{s}}$ by either 5 or 10 as indicated in the caption to Table S1. For example, in Table S1, the multipass algorithm for the PIP7 basis for $\mathrm{H}+\mathrm{H}_{2} \mathrm{O}$ lists two ranges, 1-150 and 155-200. This indicates that 150 sets were run from an $n_{\mathrm{s}}$ value of 1 to 150 and 10 sets were run with $n_{\mathrm{s}}$ values of 155, 160, 165, 170, 175, 180, 185, 190, 195, and 200. The multipass greedy code was run in parallel on a few cores (the method is trivially parallelized Table S1: Multipass schedules for reactive cases

$\mathrm{H}+\mathrm{H}_{2} \mathrm{O}, M_{\mathrm{d}}=5449$

\begin{tabular}{|c|c|c|c|}
\hline order & $N_{\mathrm{p}}$ & $n_{\mathrm{p}}$ & $n_{\mathrm{s}}{ }^{\mathrm{a}}$ \\
\hline 6 & 181 & 100 & $1-150$ \\
\hline 7 & 327 & 100 & $1-150,155-200$ \\
\hline 8 & 562 & 100 & $1-150,155-250,260-360$ \\
\hline 9 & 924 & 100 & $1-150,155-200,200-270$ \\
\hline 10 & 1461 & 100 & $1-150,155-250$ \\
\hline 11 & 2235 & 100 & $1-150,155-250$ \\
\hline
\end{tabular}

$\mathrm{H}+\mathrm{H}_{2} \mathrm{O}, M_{\mathrm{d}}=2725$

\begin{tabular}{|c|c|c|c|}
\hline order & $N_{\mathrm{p}}$ & $n_{\mathrm{p}}$ & $n_{\mathrm{s}}$ \\
\hline 10 & 1461 & 100 & $1-150,155-250$ \\
\hline
\end{tabular}

$\mathrm{H}+\mathrm{CH}_{4}, M_{\mathrm{d}}=5339$

\begin{tabular}{|c|c|c|c|}
\hline order & $N_{\mathrm{p}}$ & $n_{\mathrm{p}}$ & $n_{\mathrm{s}}$ \\
\hline 5 & 224 & 100 & $1-150$ \\
\hline 7 & 1933 & 100 & $1-150,155-170$ \\
\hline
\end{tabular}

$\mathrm{H}+\mathrm{C}_{2} \mathrm{H}_{6}, M_{\mathrm{d}}=5000$

\begin{tabular}{|c|c|c|c|}
\hline order & $N_{\mathrm{p}}$ & $n_{\mathrm{p}}$ & $n_{\mathrm{s}}$ \\
\hline 5 & 610 & 100 & $1-150$ \\
\hline 6 & 2455 & 100 & $1-150,155-250,260-280$ \\
\hline 7 & 10,010 & 100 & $1-150,155-250,260-280$ \\
\hline
\end{tabular}

${ }^{a}$ The first range is done one at a time, so that 1-150 indicates that 150 different values of $n_{\mathrm{s}}$ were used. The second range was done 5 at time $(155,160, \ldots)$. The third range was done 10 at a time $(260,270, \ldots)$

over $n_{\mathrm{s}}$ ), and calculations typically took a few hours. 
Table S2: Multipass Schedules for Non-Reactive Cases

$\mathrm{Ar}+\mathrm{NH}_{3}, M_{\mathrm{d}}=14000$

\begin{tabular}{|c|c|c|c|}
\hline order & $N_{\mathrm{p}}$ & $n_{\mathrm{p}}$ & $n_{\mathrm{s}}{ }^{\mathrm{a}}$ \\
\hline 6 & 1408 & 100 & $1-150$ \\
\hline
\end{tabular}

$\mathrm{N}_{2}+\mathrm{NH}_{3}, M_{\mathrm{d}}=14000$

\begin{tabular}{|c|c|c|c|}
\hline order & $N_{\mathrm{p}}$ & $n_{\mathrm{p}}$ & $n_{\mathrm{s}}$ \\
\hline 5 & 1638 & 100 & $1-150$ \\
\hline
\end{tabular}

$\mathrm{CH}_{4}+\mathrm{NH}_{3}, M_{\mathrm{d}}=8000$

\begin{tabular}{|c|c|c|c|}
\hline order & $N_{\mathrm{p}}$ & $n_{\mathrm{p}}$ & $n_{\mathrm{s}}$ \\
\hline 4 & 2389 & 100 & $1-150$ \\
\hline
\end{tabular}

${ }^{\mathrm{a}} 150$ different values of $\mathrm{n}_{\mathrm{s}}$ were used starting at 1 and ending with 150 .

It is clear from Tables $\mathrm{S} 1$ and $\mathrm{S} 2$ that some calculations had $N_{\mathrm{p}}$ values that were rather large compared to $M_{\mathrm{d}}$, the number of points used in the fit. However, the calculations were run in such a manner that the value of $N_{\mathrm{d}}$ was never larger than $n_{\mathrm{s}}$, as explained in the main text, so that $N_{\mathrm{d}}$ was generally much smaller than $M_{\mathrm{d}}$.

The results presented in Fig. 2 of the paper indicate that the minimum-error subset of size 56 generated from $1 \times 10^{9}$ randomly generated subsets had an rms error of $3.1 \mathrm{kcal} / \mathrm{mol}$. As indicated in Fig. 3a of the paper the forward, backward, and multipass greedy algorithms generate minimum-error subsets that have much lower rms errors than that. In fact, the multipass algorithm generates a subset of size 56 with $\mathrm{rms}$ error of $0.9972 \mathrm{kcal} / \mathrm{mol}$, rounded to $1.0 \mathrm{kcal} / \mathrm{mol}$ in the discussion in the paper (see Fig. 2). As noted in the paper it is not surprising 
that a random selection would be so inaccurate as there are $10^{103}$ unique subsets of size 56 for the PIP10 basis set whose size is 1461 .

Because there are many possible subsets, we expect that there are subsets with smaller rms errors than the ones generated with the multipass greedy algorithm. The sizes of the subsets selected by the multipass algorithm with acceptable errors are quite satisfactory for our purposes, so we are not overly concerned by this. However, we did investigate the space of subsets further and explored other options. The rest of this document summarizes some of our findings.

Our first set of investigations involved small subsets. The size of small subsets is such that we can enumerate all of them and find the subset with minimum rms error, particularly for smaller basis sets. For example, a PIP6 basis set has 181 basis functions and there are $1.5 \times 10^{9}$ subsets of size 5 , which is vastly smaller than $10^{103}$, noted above for subsets of size 56 for a PIP10 basis set. We found that the multipass greedy algorithm generally found those and in the few cases it did not, the rms error of the subset generated from the greedy algorithm had an error that was close to the one with the minimum error. Figure S1 shows two examples of our investigations, one for the PIP6 basis set for $\mathrm{H}+\mathrm{H}_{2} \mathrm{O}$ and the other for the PIP5 basis for $\mathrm{H}+$ $\mathrm{CH}_{4}$. There are eight comparisons in Fig. S1. In six cases the results of the multipass algorithm agree with the exact all-subsets calculation. For $\mathrm{H}+\mathrm{H}_{2} \mathrm{O}$ the multipass algorithm chooses a subset at $N_{\mathrm{d}}=2$ with $\mathrm{rms}$ error of $11.08 \mathrm{kcal} / \mathrm{mol}$, whereas the all-subsets calculation generates a subset with $\mathrm{rms}$ error of $11.02 \mathrm{kcal} / \mathrm{mol}$. For $\mathrm{H}+\mathrm{CH}_{4}$ the multipass algorithm chooses a subset at $N_{\mathrm{d}}=5$ with $\mathrm{rms}$ error of $5.71 \mathrm{kcal} / \mathrm{mol}$, whereas the all-subsets calculation generates a subset with rms error of $5.67 \mathrm{kcal} / \mathrm{mol}$. 


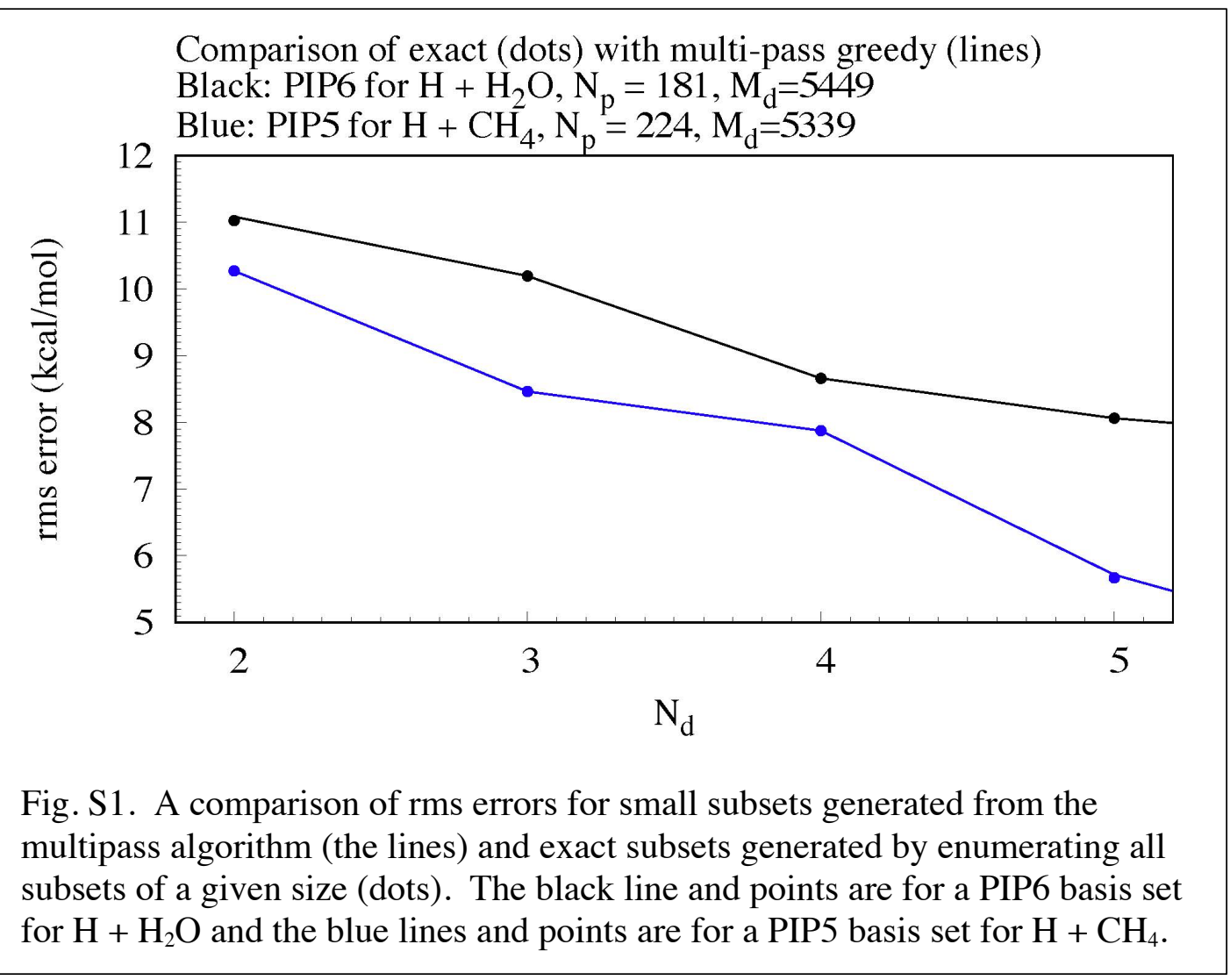

Although we consider the results in Fig. S1 to be encouraging, the space for larger subsets, ones which meet the error criteria listed in the paper, is vast. So, it seems unlikely that the multipass algorithm generates the absolute minimum subset under most conditions.

However, based on our experience with small subsets, we expect that the error is close to the minimum error. As the paper shows, the sizes of the subsets generated from the multipass algorithm within our error criteria is quite reasonable, even if there may be smaller subsets with similar rms errors.

Although all-subsets regression is out of reach for larger, lower error subsets, we can investigate the subset landscape in the vicinity of any multipass subset by exchanges. The $N_{\mathrm{d}}=$ 56 subset for $\mathrm{H}+\mathrm{H}_{2} \mathrm{O}$ investigated in Figs. 2 and 3 of the paper is selected from an $N_{\mathrm{p}}=1461$ PIP10 basis set. Fig. S2 shows the results for all the subsets generated by a complete set of 


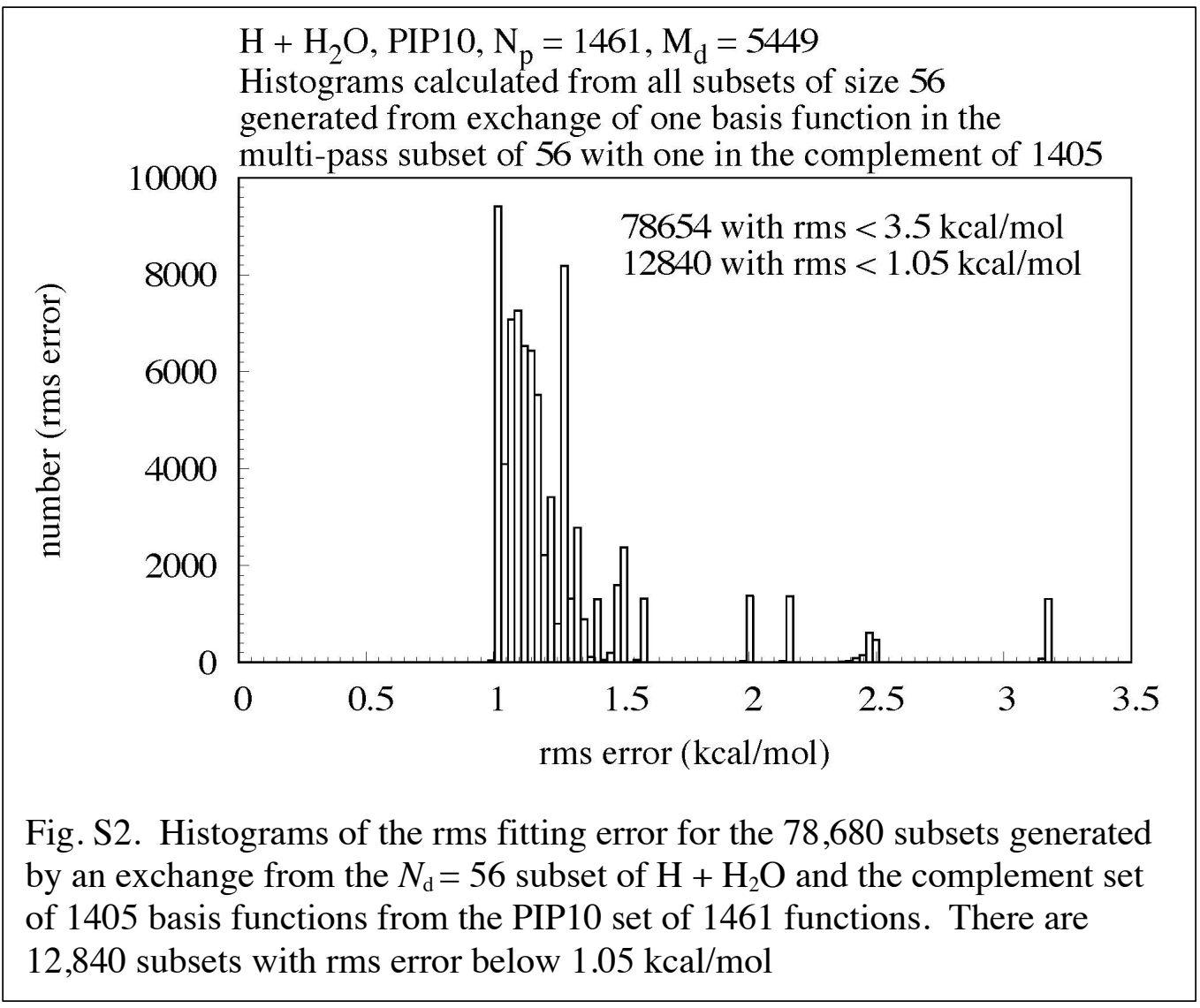

single exchanges with the other 1405 basis functions of the PIP10 basis set. A total of 78,680

unique subsets is generated by this procedure (56 x 1405). The histograms in Fig. S2 show how the rms errors of these subsets are distributed. Many of these have errors close to $1.0 \mathrm{kcal} / \mathrm{mol}$ with 12,840 having errors below $1.05 \mathrm{kcal} / \mathrm{mol}$. None of the subsets have errors below the original subset (the first bin in Fig. S2 ranges from 0.978 to $1.004 \mathrm{kcal} / \mathrm{mol}$ ). Figure S2 suggests that the landscape near the minimum multipass subset is rather complicated.

A larger set of subsets near the multipass subset can be generated from all the passes for the PIP10 dictionary (see Table S1). The full schedule of passes yields 65 unique subsets with errors below $1.05 \mathrm{kcal} / \mathrm{mol}$ and 686 unique subsets with rms errors less than $1.1 \mathrm{kcal} / \mathrm{mol}$. Exchanges were made with the set of 686 to generate $53,974,480$ subsets $(686 \times 56 \times 1405)$. These subsets, along with the original 65 , generated 247,813 unique subsets with rms errors below $1.05 \mathrm{kcal} / \mathrm{mol}$. A set of histograms summarizes this calculation in Fig. S3. There is a 


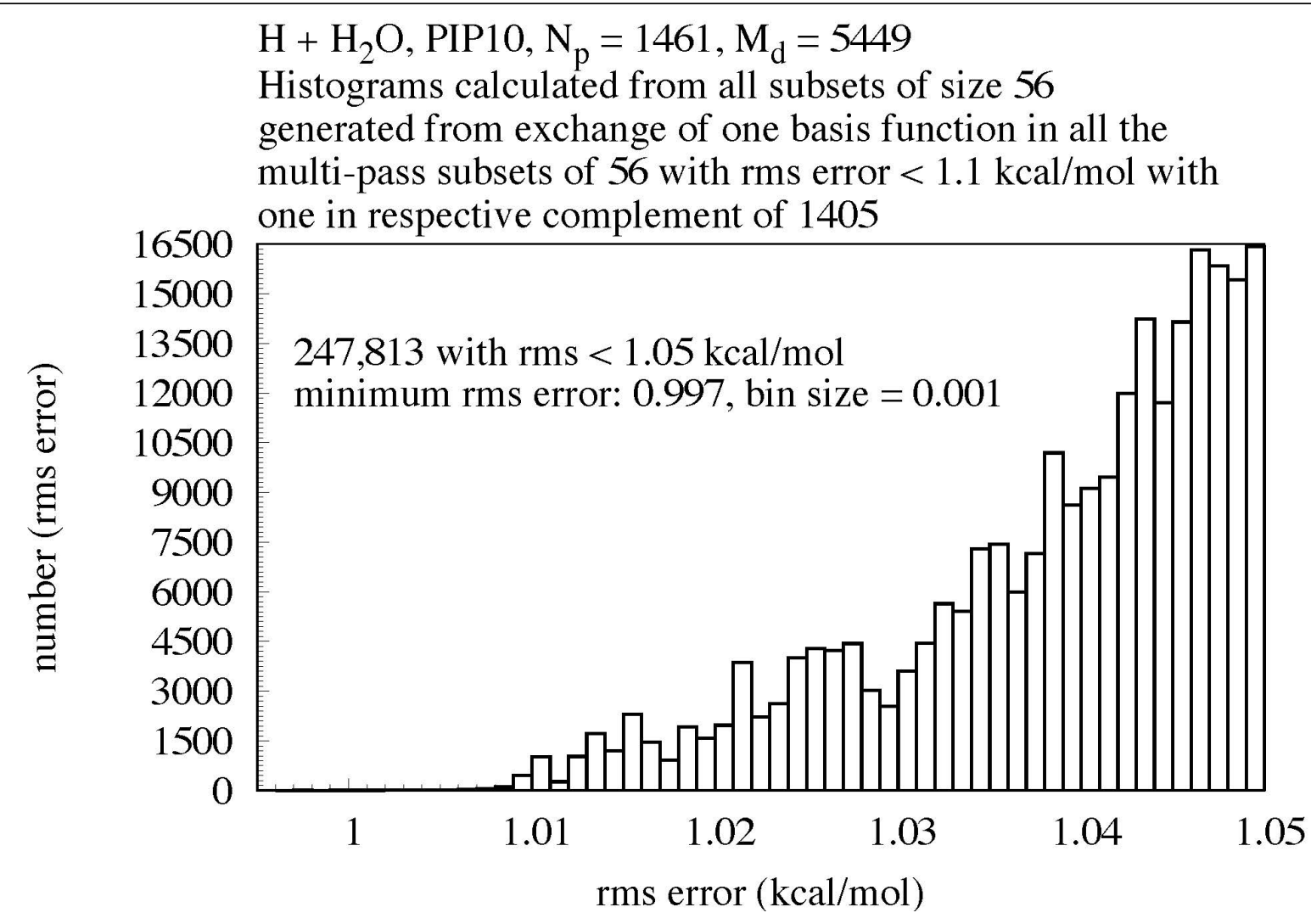

Fig. S3. Histograms for all unique $N_{\mathrm{d}}=56$ subsets with $\mathrm{rms}$ error $<1.05 \mathrm{kcal} / \mathrm{mol}$ which were generated from the subsets accumulated from all passes with a PIP10 for $\mathrm{H}+\mathrm{H}_{2} \mathrm{O}$ using single exchanges. There are 247,813 subsets with rms error less than $1.05 \mathrm{kccal} / \mathrm{mol}$. The text has further discussions.

single subset that has rms error below the multipass subset with $N_{\mathrm{d}}=56$ and the rms error of the new one is very close. Its rms error is $0.9969 \mathrm{kcal} / \mathrm{mol}$ compared to $0.9972 \mathrm{kcal} / \mathrm{mol}$ for the multipass subset. Our experience so far is that exchanges like the ones done in this document do not give a subset with a significantly lower rms error.

Figures S2 and S3 suggest that the subset landscape is quite complicated, providing an incentive to explore other ways of generating subsets. In recent years there has been an increasing interest in using mixed-integer optimization techniques to find subsets, which has been motivated to a large extent by Ref. 4. A recent issue of the journal Statistical Science focused on the utility of such methods. Two papers ${ }^{5,6}$ were published that make comparisons between this approach and sparse regression methods, accompanied by several discussion 
papers, ${ }^{7-10}$ which comment on the papers and suggest other approaches, followed by rejoinders from the authors. ${ }^{11,12}$ Mixed-integer optimization methods have been used on cases where $N_{\mathrm{p}}$ is large (see, also Ref. 13), so they may be useful for cases like the ones studied in the paper. Based on Figs. S2 and S3 the cases studied here may be more complicated than those studied in Refs. 4, 5, and 13. However, we plan on trying these methods in the future on some of the cases studied in the paper.

\section{A test of vibrational frequency convergence for the optimized PIP expansions}

Harmonic frequencies were calculated for the reactant $\mathrm{H}_{2} \mathrm{O}$ using several total order $\mathrm{H}+$ $\mathrm{H}_{2} \mathrm{O}=\mathrm{H}_{2}+\mathrm{OH}$ PIP expansions and several multipass greedy optimized expansions using the PIP11 dictionary. The relative mean unsigned error (RMUE) in the three vibrational frequencies relative to those computed at the same ab initio level of theory as the training data $(1638,3838$, and $3945 \mathrm{~cm}^{-1}$ computed using multireference perturbation theory, CASPT2/aug-cc-pVTZ, as described previously $\left.{ }^{14}\right)$ are plotted as a function of the number of adjustable parameters $\left(N_{\mathrm{d}}\right.$ for the optimized expansions and $N_{\mathrm{p}}$ for the total order expansions) in Figure S4. The RMUEs in the vibrational frequencies vary from $<1 \%$ for the largest expansions considered for each series up to just $15 \%$ for all but the smallest total-order PIP expansion, PIP3, which has an RMUE of 35\%.

The RMUEs in the vibrational frequencies are compared in Figure S4 with relative deviations in the rate constants computed from the data in Fig. 5 of the paper and using the largest total-order PIP expansion (PIP11) as the reference value. For "converged" expansions with relative deviations in the rate constant of less than $\sim 10 \%$, the errors in the vibrational frequencies are seen to be of this same order. For smaller basis sets, however, the relative errors in the rate constants are much larger than the RMUEs in the vibrational frequencies. This is 
likely a result of the more global dependence of the kinetics on the quality of the PIP expansion than one set of vibrational frequencies. Finally, we note that the sampling strategy and weight function for the training data used for this test were chosen to be appropriate for the kinetics application. Difference choices would likely be preferred (e.g., relatively larger weights near the bottom of the well) if the goal was to generate a useful PIP expansion for, say, a low-energy spectroscopy study of $\mathrm{H}_{2} \mathrm{O}$.

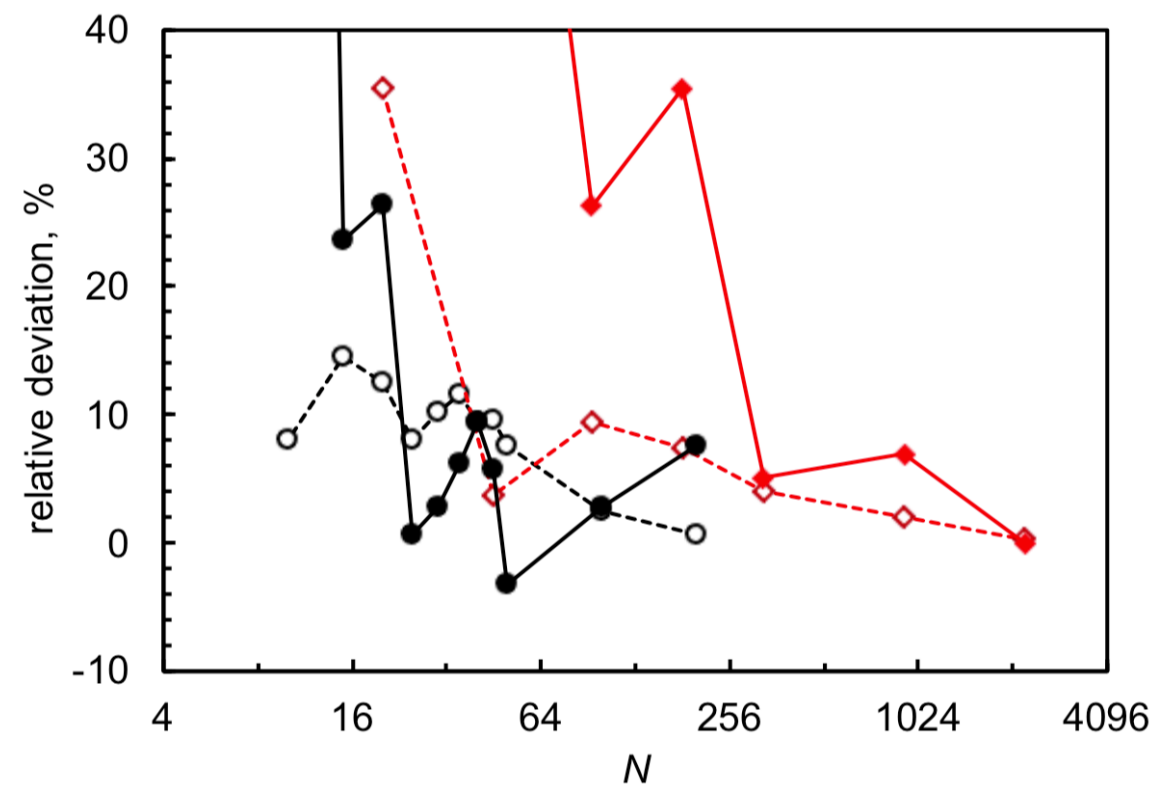

Fig. S4. Relative mean unsigned errors for the three vibrational frequencies of water (dashed lines and open symbols) compared with mean signed deviations in the rate constants reported in Fig. 5 of the paper (solid lines and closed symbols). Results are shown as a function of the number of adjustable parameters $N$ for a series of total-order PIP expansions (red diamonds, with results shown for PIP3, PIP4, PIP5, PIP6, PIP7, PIP9, and PIP11 with $N=N_{\mathrm{p}}$ varying from 20 to 2235) and a series of multipass greedy optimized PIP expansions (black circles with $N=N_{\mathrm{d}}$ varying from 10 to 200). 


\section{References for the supporting information}

${ }^{1}$ Hoaglin, D. C.; Welsch, R. E. The Hat Matrix in Regression and ANOVA Amer. Stat. 1978, $32,17-22$.

${ }^{2}$ Belsley, D. A.; Kuh, E.: Welsch R. E. Regression Diagnostics: Identifying Influential Data and Sources of Collinearity; Wiley: 1980.

${ }^{3}$ Davis M. J.; Liu W.; Sivaramakrishnan, R. Global Sensitivity Analysis with Small Sample Sizes: Ordinary Least Squares Approach. J. Phys. Chem. A 2017, 121, 553-570.

${ }^{4}$ Bertsimas, D.; King, A.; Mazumder, R. Best Subset Selection Via a Modern Optimization Lens. The Annals of Statistics 2016, 44, 813-852.

${ }^{5}$ Bertsimas, D.; Pauphilet, J.; Van Parys, B. Sparse regression: Scalable algorithms and empirical performance. Statistical Science 2020, 35, 555-578.

${ }^{6}$ Hastie, T.; Tibshirani, R.; Tibshirani, R. Best Subset, Forward Stepwise or Lasso? Analysis and Recommendations Based on Extensive Comparisons. Statistical Science 2020, 35, 579592.

7 Sarwar, O.; Sauk, B.; Sahinidis, N. V. A Discussion on Practical Considerations with Sparse Regression Methodologies. Statistical Science 2020, 35, 593-601.

${ }^{8}$ Mazumder R. Discussion of "Best Subset, Forward Stepwise or Lasso? Analysis and Recommendations Based on Extensive Comparisons. Statistical Science 2020, 35, 602-608.

${ }^{9}$ George, E. I. Modern Variable Selection in Action: Comment on the Papers by HTT and BPV. Statistical Science 2020, 35, 609-613

${ }^{10}$ Chen, Y.; Taeb, A.; Buhlmann P. A Look at Robustness and Stability of $l_{1}$-versus $l_{0^{-}}$ Regularization: Discussion of Papers by Bertsimas et al. and Hastie et al. Statistical Science 2020, 35, 614-622

${ }^{11}$ Bertsimas, D.; Pauphilet, J.; Van Parys, B. Rejoinder: Sparse Regression: Scalable Algorithms and Empirical Performance. Statistical Science 2020, 35, 623-624.

${ }^{12}$ Hastie, T.; Tibshirani, R.; Tibshirani. Rejoinder: Best Subset, Forward Stepwise or Lasso? Analysis and Recommendations Based on Extensive Comparisons. Statistical Science 2020, $35,625-626$

${ }^{13}$ Bertsimas, D.; Van Parys, B. Sparse High-Dimensional Regression: Exact Scalable Algorithms and Phase Transitions. The Annals of Statistics 2020, 48, 300-323. 
${ }^{14}$ Tao, Y.; Jasper, A. W.; Georgievskii, Y.; Klippenstein, S. J.; Sivaramakrishnan, R. Termolecular Chemistry Facilitated by Radical-Radical Recombinations and its Impact on Flame Speed Predictions. Proc. Combust. Inst. 2021, 31, 515-522. 